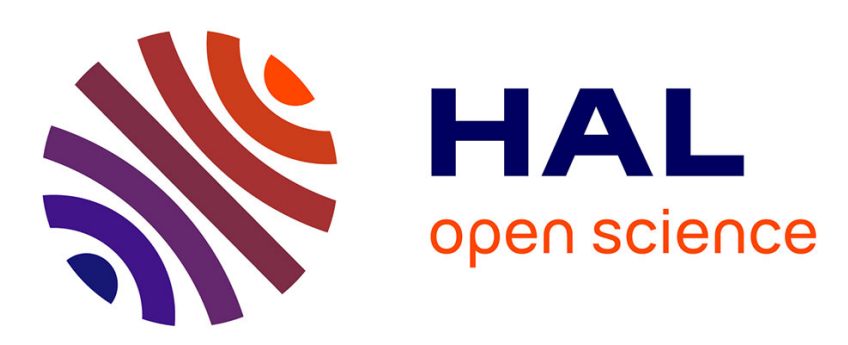

\title{
The electrical behavior of superconducting thin-film microbridges self-heating and superconducting quantum processes
}

W.J. Skocpol, M. R. Beasley, M. Tinkham

\section{- To cite this version:}

W.J. Skocpol, M. R. Beasley, M. Tinkham. The electrical behavior of superconducting thin-film microbridges self-heating and superconducting quantum processes. Revue de Physique Appliquée, 1974, 9 (1), pp.19-23. 10.1051/rphysap:019740090101900 . jpa-00243739

HAL Id: jpa-00243739

https://hal.science/jpa-00243739

Submitted on 1 Jan 1974

HAL is a multi-disciplinary open access archive for the deposit and dissemination of scientific research documents, whether they are published or not. The documents may come from teaching and research institutions in France or abroad, or from public or private research centers.
L'archive ouverte pluridisciplinaire $\mathbf{H A L}$, est destinée au dépôt et à la diffusion de documents scientifiques de niveau recherche, publiés ou non, émanant des établissements d'enseignement et de recherche français ou étrangers, des laboratoires publics ou privés. 


\title{
THE ELECTRICAL BEHAVIOR OF SUPERCONDUCTING THIN-FILM MICROBRIDGES \\ SELF-HEATING AND SUPERCONDUCTING QUANTUM PROCESSES (*)
}

\author{
W. J. SKOCPOL ${ }^{\dagger}, M$. R. BEASLEY and M. TINKHAM \\ Department of Physics and Division of Engineering and Applied Physics \\ Harvard University, Cambridge, Massachusetts 02138, USA
}

\begin{abstract}
Résumé. - Nous présentons les mesures des caractéristiques $I-V$ de microponts supraconducteurs en couches minces et de ces mesures nous déduisons quelques nouveaux résultats concernant le comportement électrique de ces microponts. A basse température, nous trouvons que leur comportement est dominé par les effets d'échauffement. Pour les faibles valeurs de la tension et près de $T_{\mathrm{c}}$ où l'échauffement n'est pas important, nous trouvons que le comportement observé est très bien décrit par un modèle de glissement de phase et que les courants normaux qui circulent pendant le processus de glissement de phase ont une extension spatiale déterminée par la longueur de diffusion des quasi-particules. Même près de $T_{\mathrm{c}}$ l'échauffement devient important lorsque les tensions sont élevées; il est probable que ceci limite la tension maximale (et de là la fréquence Josephson) jusqu'à laquelle le comportement de type Josephson peut être obtenu.
\end{abstract}

\begin{abstract}
Measurements of the $I-V$ curves of superconducting thin-film microbridges are reported and used to infer some new results regarding the electrical behavior of these devices. At temperatures well below $T_{\mathrm{c}}$ their behavior is found to be dominated by the effects of self-heating. At low voltages near $T_{\mathrm{c}}$ where heating is not important, it is found that the observed behavior is best understood in terms of a phase-slip mechanism and that the normal currents which flow during the phase-slip process extend over a range determined by the quasiparticle diffusion length. Even near $T_{\mathrm{c}}$ heating becomes important at high voltages and probably puts a limit on the maximum voltage (hence Josephson frequency) up to which useful Josephson-like behavior can be obtained.
\end{abstract}

1. Introduction. - Recent experimental [1]-[3] and theoretical [4]-[5] work has shown that superconducting thin-film microbridges which are small compared to the Ginzburg-Landau (GL) coherence length $\xi(T)$ (i. e., very near $T_{\mathrm{c}}$ ) apparently exhibit classical Josephson behavior : a sinusoidal current-phase relationship, a linear temperature dependence of the critical current, and an ac Josephson effect with a quasi-Bessel function dependence on microwave power. By comparison, considerably less attention has been paid recently to the regime further below $T_{\mathrm{c}}$, where for convenience in making practical devices one would hope useful Josephson behavior might be obtained, even if it is not of the classical form. We have recently undertaken a thorough study of the current-voltage $(I-V)$ characteristics of a variety of microbridges (both long and short) over a wide range of temperatures. In this paper we attempt to summarize our major results and what they reveal about the underlying physical processes governing the electrical behavior of these devices.

In brief, our results show that well below $T_{\mathrm{c}}$ the

$\left(^{*}\right)$ Work supported in part by the Office of Naval Research, the National Science Foundation, and the Joint Services Electronics Program.

† Danforth Fellow. electrical behavior of these microbridges is largely dominated by the effects of self-heating. Only at low voltage (hence low power) levels near $T_{\mathrm{c}}$ is the behavior found to be solely governed by superconducting quantum processes. Moreover, in this intrinsic superconducting regime, the observed $I-V$ curves can best be understood in terms of a phase-slip model like that introduced originally by Notarys and Mercereau [6] to describe the behavior of proximity effect bridges, rather than the popular shunted-Josephson-junction model [7], which has been used frequently in the past to interpret the behavior of superconducting microbridges. However, in contrast to the theory of the phase-slip process recently developed by Rieger, Scalapino and Mercereau (RSM) [8], we find that in the tin microbridges we have studied, the normal current flow during the phase-slip process extends over a length determined by the quasiparticle diffusion length of Pippard, Shepherd and Tindall [9], and not the Ginzburg-Landau coherence length as found by RSM. In what follows we present the evidence for these conclusions and discuss their implications for applications of these devices. In particular, our results indicate that self-heating probably plays a role in limiting the maximum voltage (hence Josephson frequency) up to which these devices can exhibit useful Josephson-like behavior. 
2. Self-heating effects. - Figure 1 shows the $I-V$ curves for typical long and short tin microbridges at various helium bath temperatures. The evidence for strong self-heating effects is immediately evident. In both cases the $I-V$ curves show pronounced anomalies due to the onset of bubble nucleation at the sample surface and a substantial change in the $I-V$ curve upon cooling through the superfluid transition. Both changes result in improved heat transfer. The identification of the bubbling anomalies is confirmed by their absence both below the superfluid transition and in a vacuum environment. Further evidence for heating is obtained from the generally good fit to the observed $I-V$ curves obtained from a simple theory of self-heating (dotted curves) in which resistance is assumed to arise from a

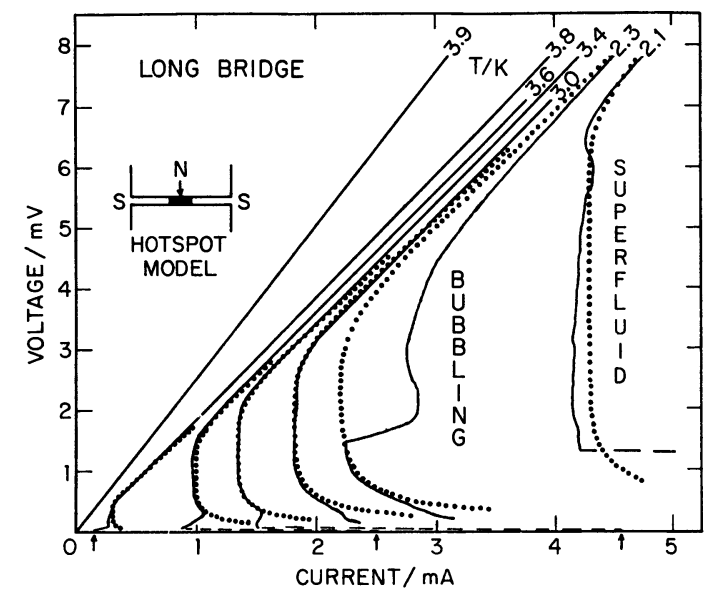

(a)

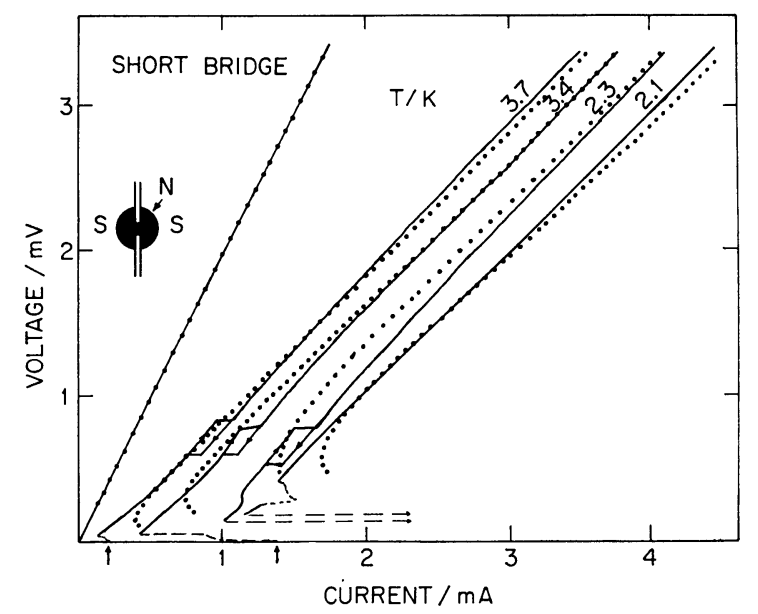

(b)

FIG. 1. $-I-V$ characteristics for a long $(40 \mu \mathrm{m}$ by $3.0 \mu \mathrm{m}$ by $0.1 \mu)$ microbridge and a short $(\sim 0.5 \mu \mathrm{m}$ by $\sim 0.5 \mu \mathrm{m}$ by $0.1 \mu \mathrm{m})$ microbridge directly immersed in liquid helium for a series of bath temperatures. The solid and dashed curves are experimental data; the dashed curves are the dc average of a circuit-controlled relaxation oscillation at low bias voltages. The arrows along the current axis indicate the critical currents $I_{\mathrm{c}}$. The effects of heating are obvious from the anomalies due to bubbling at the sample surface, and the superfluid transition, both of which increase the heat transfer. The dotted curves are theoretical results obtained from a simple model of a self-heating normal hotspot (described in the text). The inserts show the relative size of a typical hotspot compared to that of the bridge. fully normal hot-spot maintained above $T_{\mathrm{c}}$ by Joule heating. The deviations at low voltages are due to resistive superconducting behavior and are discussed in section 3 . The dashed part of the measured $I-V$ curves indicates the dc average of circuit-controlled relaxation oscillations observed for voltage biasing in this region. When current biased the bridges show hysteretic switching at $I_{\mathrm{c}}$ and at the minimum current reached on each curve.

In the self-heated hostpot theory, which will be discussed in detail in a subsequent paper [10], heat is generated in a fully normal region (see inserts in figure 1) by an applied current $I$ and is carried away both through conduction and heat transfer across the boundaries with the helium bath and sample substrate. The size of the normal region and hence the voltage for the given current is determined self-consistently by the condition that the resulting temperature distribution has $T=T_{\mathrm{c}}$ at the superconducting/normal interface. For long bridges it is sufficient to assume that the temperature is maintained at the bath temperature at the ends of the bridge. For short bridges it is essential to at least approximate the spread of the normal region into the wide film at the ends of the bridge. The results of our analysis are analytic expressions from which the theoretical $I-V$ curves of figure 1 were obtained. In calculating these curves, most of the parameters (e. g., bridge dimensions, the normal resistivity $\rho$, and $T_{\mathrm{c}}$ ) were independently determined; only the thermal conductivity $\kappa$ and the heat transfer coefficient $\alpha$ were unknown and treated as adjustable parameters. The fitted values of $\kappa$ are consistent with those found from $\rho$ using the Wiedemann-Franz law. The fitted values of $\alpha$ fall in the range $1.0-3.5 \mathrm{~W} / \mathrm{cm}^{2} / \mathrm{K}$ for transfer to the substrate and/or substrate plus normal helium bath, and 7-9 W/ $\mathrm{cm}^{2} / \mathrm{K}$ for transfer to the substrate plus superfluid helium; this is in good agreement with published values. The calculated curves for short bridges are relatively insensitive to $\alpha$ because most of the heat is carried away from the normal region by conduction to the strong cooling area provided by the wide part of the film at the ends of the bridge. For the same reason, they are substantially better cooled than the long bridges. While the details of both the observed $I-V$ curves (e. g., the region of negative differential resistance) and their interpretation as a result of heating are quite interesting, our objective here is only to establish the importance of self-heating in these devices and to demonstrate that at low temperatures and high voltages the heating effects can be satisfactorily understood with a simple model of a normal hotspot. A complete discussion of these effects and the theory will appear in our subsequent paper.

3. Superconducting quantum processes. - As is evident in figure 1, at low voltages the data systematically deviate from the predictions of the normal hotspot model. In this regime superconducting resis- 
tive processes play a role in determining the $I-V$ curve. As $T \rightarrow T_{\mathrm{c}}$ the importance of self-heating diminishes and the behavior becomes governed by these intrinsic superconducting processes. This diminution of the importance of self-heating as $T \rightarrow T_{\mathrm{c}}$ can be seen from the fact that the minimum current necessary to maintain a fully normal hotspot $I_{\min }^{\text {hs }}$ (i. e., the current at the point of infinite differential resistance in the theoretical curves of figure 1$)$ decreases only as $\left(T_{\mathrm{c}}-T\right)^{1 / 2}$, whereas the critical current, following the GL meanfield behavior, decreases as $\left(T_{\mathrm{c}}-T\right)^{3 / 2}$. Thus at some temperature near $T_{\mathrm{c}}, I_{\mathrm{c}}$ becomes less than $I_{\min }^{\mathrm{hs}}$ and superconducting resistive behavior unaffected by heating has an opportunity to develop. This relationship between $I_{\mathrm{c}}$ and $I_{\min }^{\mathrm{hs}}$ is clearly evident in figure 1 .

The low voltage behavior near $T_{\mathrm{c}}$ of the short bridge of figure 1 is shown more fully and enlarged in figure 2 . As is evident from this figure, at low voltage the $I-V$ curves are very simple : as $T \rightarrow T_{\mathrm{c}}$, they are characterized by a temperature-independent differential resistance $\mathrm{d} V / \mathrm{d} I$ and an " excess " average supercurrent of $\approx \frac{1}{2} I_{\mathrm{c}}$. (At lower bath temperatures the excess supercurrent is apparently less than $\frac{1}{2} I_{\mathrm{c}}$.) At high voltages the $I-V$ characteristics gradually curve toward the voltage axis and smoothly join the heating dominated part of the characteristic.

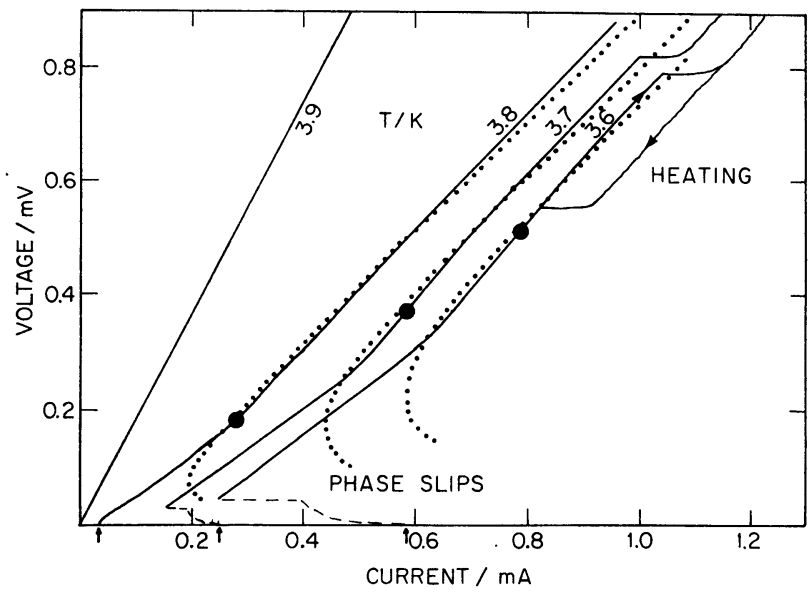

Fig. 2. - I-V characteristics near $T_{\mathrm{c}}$ for the short bridge of figure 1, showing the heating effects at high voltages and the phase-slip behavior at low voltages. The large dots indicate the voltage $2 \Delta(T) / \mathrm{e}$

The form of the $I-V$ curves in figure 2 is very similar to that observed in proximity effect junctions, and can be qualitatively understood in terms of a phase-slip model originally introduced by Notarys and Mercereau [6]. In this model the phase slippage which is required at finite voltages by the Josephson equation $V=(\hbar / 2 \mathrm{e}) \mathrm{d} \varphi / \mathrm{d} t$ arises from a relaxation oscillation of the order parameter. A qualitative picture of this model is as follows. Because for $I>I_{\mathrm{c}}$ some of the current is carried by the normal electrons, on the average there must be a potential gradient along the bridge. An important consequence of this potential gradient is that the superfluid velocity $v_{\mathrm{s}}$, which is assumed to behave nearly as in a one-dimensional superconductor, cannot be constant in time but must accelerate. However, once the supercurrent density $J_{\mathrm{s}}=2 \mathrm{e}|\psi|^{2} v_{\mathrm{s}}$ reaches $J_{\mathrm{c}}$, the order parameter $\psi$ becomes unstable and rapidly collapses to zero, forcing the supercurrent (but not $v_{s}$ ) to zero also and necessitating a shift of all the current into the normal channel. The end result of this instability is a region (presumably $\sim \xi(T)$ in length) in which $\psi \approx 0, J_{\mathrm{s}} \approx 0$, but across which a phase difference $\Delta \varphi \approx 2 \pi$ exists. At this point the system is assumed to " snap back" to a state where $\Delta \varphi$ has decreased by exactly $2 \pi$ and now $\psi, J_{\mathrm{s}}$, and $\Delta \varphi$ all are approximately equal to zero. At this point superconductivity can reform and the supercurrent reaccelerate. The resulting behavior is a periodic increase and decrease of the supercurrent density (at the Josephson frequency) between $J_{\mathrm{s}}=0$ and $J_{\mathrm{s}}=J_{\mathrm{c}}$ coupled with a growth and decay of the amplitude of the order parameter, all of which culminates in an abrupt phase slip of $2 \pi$ at the end of each cycle. The dc $I-V$ curve that results from this process is given by the expression

$$
\bar{V}=R_{\mathrm{n}}\left(I-\overline{I_{\mathrm{s}}(t)}\right)
$$

where $R_{\mathrm{n}}$ is the normal resistance of the region over which the normal currents flow during the phase slip process. The $I-V$ curves shown in figure 2 are just of this form where $\overline{I_{\mathrm{s}}(t)} \approx \frac{1}{2} I_{\mathrm{c}}$ and $R_{\mathrm{n}}$ is temperature independent. Moreover, as seen from the figure, once started the phase-slip process can persist even for $I<I_{\mathrm{c}}$.

The details of the phase-slip process are obviously quite subtle, involving not only complicated superfluid dynamics, but also delicate issues such as non equilibrium between the normal and superfluids as well. Two relatively simple attempts to provide a more detailed theoretical description of this process using time-dependent Ginzburg-Landau theory to describe the superfluid dynamics have been undertaken : one in the paper by RSM and the second more recently by Tinkham [11]. In both of these calculations $\overline{I_{\mathrm{s}}(t)}$ is found to be $\approx \frac{1}{2} I_{\mathrm{c}}$ in good agreement with our results. However, these two theories differ substantially in their assumptions regarding the characteristic healing length for departures from mutual equilibrium of the normal and superfluid components of the superconductor, and consequently lead to different predictions for $R_{\mathrm{n}}$. We will return to this issue below.

We have found that at low voltages near $T_{\mathrm{c}}$ long microbridges also exhibit phase slippage at localized voltage centers and that this provides the basis of an explanation of the well-known voltage steps observed in the $I-V$ characteristics of long superconducting microbridges and whisker crystals [12], [13]. Many steps occur because the length of the bridges is large compared to the size of a phase-slip center. By means of voltage tabs located along the length of some of 
our long microbridges, we have directly established that the observed steps arise from spatially localized voltage centers and that the onset current for a given step is primarily determined by local variations of $T_{\mathrm{c}}$, and hence $I_{\mathrm{c}}$, along the bridge. These variations in $T_{\mathrm{c}}$ were established by measuring $I_{\mathrm{c}}(T)$ for the various segments of the bridge defined by the voltage tabs. Figure 3 shows the individual $I-V$ curves associated with the first three voltage steps (each corresponding to a different segment of the bridge) in the total $I-V$ characteristic of a $140 \mu$ long tin microbridge. The curves shown in figure 3 were chosen so as to correspond approximately to the same temperature difference from the local $T_{\mathrm{c}}$ 's. The curves obtained from the three voltage units are virtually identical and bear a striking resemblance to those observed with the short bridge of figure 2. It seems almost certain that the spatially localized voltage centers observed in the long bridges are phase-slip centers basically like those found in short bridges. Firm confirmation of this assertion lacks only demonstration of the ac Josephson effect on one of the voltage plateaus in the $I-V$ characteristic of a long bridge or whisker.

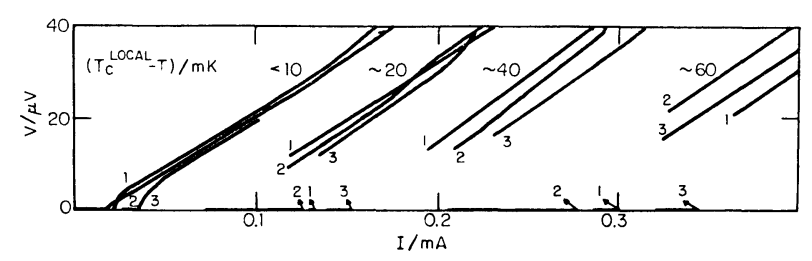

FIG. 3. - Low voltage behavior of the steps near $T_{\mathrm{c}}$ in the $I-V$ curves of a long $(140 \mu \mathrm{m}$ by $3 \mu \mathrm{m}$ by $0.1 \mu \mathrm{m})$ microbridge with voltage tabs along its length. The steps arise from phaseslip centers spatially localized in different regions of the bridge. The voltage plotted is the local voltage drop across each of the first three steps that appear. The curves presented were selected to represent approximately equal temperature differences from the local $T_{\mathrm{c}}$ 's. The curves show a temperature independent slope and average supercurrent of $\sim \frac{1}{2} I_{\mathrm{c}}$ like those shown in figure 2 , indicating that each voltage center of a long bridge acts just like a phase-slip center in a short bridge.

Note added in proof. - We have now observed the ac Josephson effect in phase-slip centers in a $120 \mu$ long, geometrically uniform bridge, as evidenced by microwave-induced steps at the voltage corresponding to the $10 \mathrm{GHz}$ microwave frequency. As in short bridges, subharmonic steps as well as the fundamental step are observed, consistent with the anharmonic current waveform predicted by the phaseslip model. When two phase-slip centers are present, the induced steps occur when the local voltage drop across either center corresponds to the microwave frequency; no induced step is seen when the total voltage across both centers corresponds to the microwave frequency.

The importance of the results on long bridges (in addition to providing an explanation of the observed voltage steps) lies in the fact that they allow a precise determination of the length of the nonequilibrium region over which normal currents flow during a phase slip. If this length is denoted by $L_{\mathrm{n}}$, then for long microbridges the resistance $R_{\mathrm{n}}$ in (1) is given by the simple expression

$$
R_{\mathrm{n}}=\frac{L_{\mathrm{n}}}{L_{\mathrm{T}}} R_{\mathrm{T}}
$$

where $L_{\mathrm{T}}$ and $R_{\mathrm{T}}$ are the total length and normal resistance of the microbridge. For short bridges $R_{\mathrm{n}}$ depends only logarithmically on $L_{\mathbf{n}}$.

Using the data on the bridge shown in figure 3 $\left(R_{\mathrm{T}}=2.4 \Omega\right.$ and $\left.L_{\mathrm{T}}=140 \mu \mathrm{m}\right)$, we find that $L_{\mathrm{n}}=14 \mu \mathrm{m}$ and is temperature independent. Data on other long bridges yields values in the range $8-16 \mu \mathrm{m}$ with the longer values generally corresponding to the bridges with the longer mean free paths $l$. Data from short bridges are consistent with these values. Using the data of Meyer and v. Minnegerode [13] for voltage steps in tin whisker crystals, we find $L_{\mathrm{n}}$ lies in the range $48-60 \mu \mathrm{m}$ for these very clean $(l \approx 2 \mu \mathrm{m})$ systems. These measured values are not consistent with the result $L_{\mathrm{n}} \approx 2 \xi(T)$ expected from the theory of RSM. First of all, the observed $L_{n}$ 's are temperature independent, and second, for the samples we have considered, they are larger than $\xi(T)$ in the temperature range under consideration.

Since $L_{\mathrm{n}}$ is temperature independent near $T_{\mathrm{c}}$ it seems natural to suppose that it is somehow related to the normal excitations, and not the superfluid. In fact our data are remarkably consistent with the identification $L_{\mathrm{n}}=2 \Lambda$, where $\Lambda=\left(l_{\mathrm{F}} \tau_{2}\right)^{1 / 2}$ is the quasiparticle diffusion length introduced by Pippard et al. [9], to account for the excess resistance observed at SNS interfaces. Here $v_{F}$ is the Fermi velocity and $\tau_{2}$ is the inelastic scattering time (including recombination) for the quasiparticles. In calculating $\Lambda$ for our bridges, we have used $v_{\mathrm{F}}=10^{8} \mathrm{~cm} / \mathrm{s}$, $\tau_{2}=4 \times 10^{-10} \mathrm{~s}[14]$, and $l=0.1 \mu \mathrm{m}$ and $2 \mu \mathrm{m}$ (as estimated from $\rho$ ) in microbridges and whiskers respectively. Physically $\Lambda$ represents the distance quasiparticles can random walk before inelastic scattering. A phenomenological model of the phaseslip process in which the healing length for departures from equilibrium between the quasiparticles and the superfluid is given by $\Lambda$ has been developed by Tinkham [11] and is in good agreement with our results. It should be noted, however, that in Tinkham's theory it has been assumed that $\Lambda \gg \xi(T)$. In the opposite limit $\Lambda \lesssim \xi(T)$, the theory of RSM may be correct.

4. Conclusions. - The results presented in this paper, along with the previous work on very small bridges near $T_{\mathrm{c}}$, allow us to draw the following tentative picture of the complete electrical behavior of superconducting thin-film microbridges. Very near $T_{\mathrm{c}}$ where $\xi(T)$ is large compared to the length $L$ of the bridge, classical Josephson behavior is apparently obtained. However, as the temperature is decreased, or if the bridge is not short enough, so 
that $\xi(T)<L$, behavior like that observed in these experiments is obtained; the bridge acts much more "bulk-like ", having a current-phase relation $I_{\mathrm{s}} \propto|\psi|^{2} \nabla \varphi$ and a critical current following the mean-field behavior $I_{\mathrm{c}} \propto\left(T_{\mathrm{c}}-T\right)^{3 / 2}$, but still exhibits Josephson-like behavior through the phaseslip mechanism. Unfortunately a completely satisfactory theory of this phase-slip process is not yet available, although some progress has been made through our work on long bridges, which has identified the important role of the quasiparticle diffusion length $\Lambda$ in the nonequilibrium aspects of the process. How long the bridge can be before single-unit Josephsonlike behavior is lost is not clear, but a plausible guess would be $L \approx 2 \Lambda$ when $\Lambda>\xi$, rather that $L \approx 2 \xi(T)$ as has been suggested previously, since as analyzed by Tinkham [11] the presence of one phase-slip center tends to stabilize adjacent regions against the formation of additional centers over a range $2 \Lambda$. Since $\Lambda$ can be quite long, this might be useful in fabricating microbridge weak links.

For temperatures far below $T_{\mathrm{c}}$, the behavior of these bridges at finite voltages appears to be almost completely dominated by the effects of self-heating, and in this region resistance is produced by a fully normal hot spot rather than by phase slippage. Even near $T_{\mathrm{c}}$, where at low voltages self-heating is not important, our results suggest that as the bias voltage is increased, self-heating does become important even at voltages less than $2 \Delta(T) / \mathrm{e}$. (See figure 2 where the voltage $2 \Delta(T) / \mathrm{e}$ is indicated by the circles.) As heating becomes important, the temperature of the bridge begins to rise and $I_{\mathrm{c}}$ and hence $\overline{I_{\mathrm{s}}(t)}$ are decreased. At higher voltages and correspondingly higher heat inputs, phase coherence is ultimately lost and a hotspot forms. The curvature of the $I-V$ curves toward the voltage axis in figure 2 appears to be indicative of just this process.

If this interpretation is correct, then clearly selfheating plays a role in limiting the maximum voltage and hence Josephson frequency up to which these devices can operate. Moreover, preliminary results of an analysis of this aspect of the heating problem suggest that self-heating should be important at voltages less than but comparable to $2 \Delta(T) / \mathrm{e}$ for small microbridges generally, regardless of the particular material used. A comparison of the importance of self-heating in various types of weak links suggests that point contacts, where substantial material is available to carry away the heat in three dimensions, should suffer much less limitation due to heating than microbridges in high frequency applications. Proximity effect bridges, on the other hand, may suffer the most severe limitations, since as they are presently constructed the junction is effectively placed at the center of a long bridge and therefore is not cooled as efficiently as short microbridges. These conclusions are clearly speculative, however, and direct experimental confirmation of this limitation would be highly desirable.

\section{References}

[1] Fulton, T. A. and Dynes, R. C., Phys. Rev. Lett. 25 (1970) 794.

[2] Gregers-Hansen, P. E. and Levinsen, M. T., Phys. Rev. Lett. 27 (1971) 847.

[3] Song, Y. and Rochlin, G. I., Phys. Rev. Lett. 29 (1972) 416.

[4] Baratoff, A., Blackburn, J. A. and Schwartz, B. B., Phys. Rev. Lett. 25 (1970) 1096.

[5] Gregers-Hansen, P. E., Levinsen, M. T. and Fog PederSEN, G., J. Low Temp. Phys. 7 (1972) 99.

[6] Notarys, H. A. and Mercereau, J. E., Physica 55 (1971) 424.

[7] MCCumber, D. E., J. Appl. Phys. 39 (1968) 3113 ; Stewart, W. C., Appl. Phys. Lett. 12 (1968) 277.
[8] Rieger, T. J., Scalapino, D. J. and Mercereau, J. E., Phys. Rev. B 6 (1972) 1734.

[9] Pippard, A. B., Shepherd, J. G. and Tindall, D. A., Proc. R. Soc. A 324 (1971) 17.

[10] Skocpol, W. J., Beasley, M. R. and Tinkham, M., Bull. Am. Phys. Soc. 18 (1973) 302, and to be published.

[11] TinkHam, M., to be published.

[12] Webb, W. W. and Warburton, R. J., Phys. Rev. Lett. 20 (1968) 461 ; also see Warburton, R. J. and WebB, W. W., Bull. Am. Phys. Soc. 13 (1968) 379.

[13] Meyer, J. and v. Minnegerode, G., Phys. Lett. A 38 (1972) 529 ; Proc. 13th Int. Conf. on Low Temp. Phys. (Boulder) 1972 to be published.

[14] Tinkham, M., Phys. Rev. B 6 (1972) 1747. 